\title{
Research Paper \\ Psychometric Properties of Body Image Psychological Inflexibility Scale
}

\author{
Sohrab Amiri ${ }^{1 *}$, Abolghasem Yaghoubi ${ }^{2}$
}

1. PhD in Psychology, Department of Psychology, Faculty of Literature and Humanities, Urmia University, Urmia, Iran.

2. PhD in Psychology, Associate Professor, Department of Psychology, Faculty of Economics and Social Sciences, Bu-Ali Sina University, Hamadan, Iran

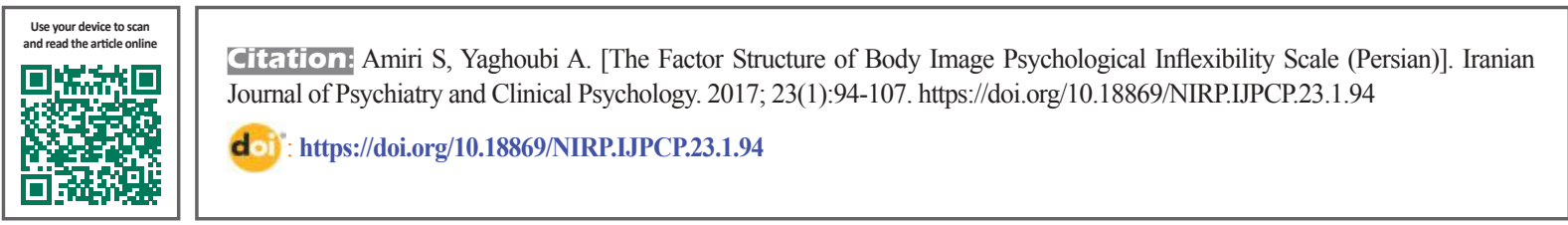

Received: 30 Oct. 2016 Accepted: 19 Feb. 2016

Key words:

Psychological inflexibility, Body image, Body dysmorphic, Factor analysis

\section{ABSTRACT}

Objectives The aim of present study was to examine the psychometric properties of the Iranian version of the Body Image Psychological Inflexibility Scale (BIPIS) in students of both genders.

Methods The study sample consisted of Bu-Ali-Sina University students enrolled during the academic year 2016. A total of 329 students were selected based on multistage cluster sampling. To study the psychometric characteristics of the BIPIS, it was first translated with the help of the double-translation technique and then administered along with the Body Dysmorphic Meta-Cognitive Questionnaire and Body Image-Acceptance and Action questionnaire. The collected data was analyzed, and thereafter, its reliability, validity and confirmatory factor analysis were calculated.

Results Analysis of the collected data showed that the scale has a reliability of 0.90 to 0.93 alpha coefficients in general and both genders. In addition, the concurrent validity of both genders with the sub-scales of Body Dysmorphic Meta-Cognitive Questionnaire and Body Image-Acceptance and Action Questionnaire was $0.71,0.62,0.75,0.69$ and -0.58 for Meta-Cognitive strategies, thought- action fusion, Meta-Cognitive negative and positive beliefs, safety behaviors, and body image-acceptance and action questionnaire respectively. Which in the $\mathrm{P}<0.01$ level was significant. Moreover, the results of the confirmatory factor analysis supported the factor structure of the questionnaire.

Conclusion The BIPIS favors psychometric properties among Iranian students.

\section{Extended Abstract}

\section{Introduction}

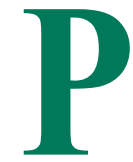

sychological flexibility includes the ability of the individual to fully experience thoughts and feelings without resistance $[13,14]$. Body image inflexibility occurs when people are reluctant to experience thoughts, emotions and physical feelings associated with body image and attempt to change the shape or the occurrence of these experiences [15]. Body image inflexibility also represents a set of behaviors characterized by the avoidance of painful and traumatic experiences that damage personal values $[13,14]$. To the best of my knowledge, no study in Iran evaluates the psychometric properties and factor structure of Body Image Psychological Inflexibility Scale (BIPIS) [18]. This study, therefore, examines the validity, reliability and factor structure of BIPIS with Iranian students as the sample population.

\section{Method}

This research used descriptive-psychometric methods. The study sample comprised students of Bu-Ali-Sina University enrolled in the academic year 2016. A total of

* Corresponding Author:

Sohrab Amiri, PhD Student

Address: Department of Psychology, Faculty of Literature and Humanities, Urmia University, Urmia, Iran.

Tel: +98 (44) 17058990

E-mail: amirysohrab@yahoo.com 
329 students were selected based on a multistage cluster sampling. To evaluate the psychometric properties of the scale, Body image inflexibility Scale was first translated with the help of the reverse translation technique and then administered along with the Body Dysmorphic Meta-Cognitive Questionnaire and Image Acceptance and Action Questionnaire. The collected data were analyzed using the SPSS software (ver. 22) and LISREL-8.8. Statistical methods such as Cronbach's alpha, criterion validity, correlation, exploratory and confirmatory factor analysis were put to use.

\section{Results}

Descriptive and demographic characteristics of the participants are presented in Table 1. Reliability: To investigate the stability of BIPIS, Cronbach's alpha coefficients, Split-Half and test-retest coefficients were calculated. Cronbach's alpha coefficient of the 16-Questions scale was 0.92 , which indicated that the scale has good internal consistency.

The results of Cronbach's alpha coefficient, Stock split up and retest are presented in Table 2. The results in Table 2 show that Cronbach's alpha coefficient was satisfactory, and the data pertaining to retest reliability and Split-Half coefficients were also significant.

Validity: Correlation coefficients for BIPIS and subscales of Meta-Cognitive physical deformities scale were 0.71 for Meta-Cognitive control strategies, 0.62 for objectification of thoughts, 0.75 for positive and negative Meta-Cognitive beliefs, 0.69 for safety behav- iors and -0.58 for Body Image Psychological Flexibility Questionnaire which significant in $\mathrm{P}<0.01$.

The pattern of correlation coefficients of sub-scales, physical deformities Meta-Cognition Scale [26] and body image flexibility questionnaire [15] represented criterion validity as well as favorable divergence validity of the Persian version of BIPIS [18]. Factor Analysis: The value obtained for Kaiser-Meyer-Olkin Measure of Sampling Adequacy was equal to 0.79 and Bartlett's test of sphericity (Chi-Square=478.38) 3 showed that factor analysis is possible. The results of the factors slope graph (scree plot) in Figure 1 and analysis of principal components showed the presence of a component with values greater than 1 , which explained 64.69 percent of the variance.

\section{Discussion}

The reliability analysis in this study showed a good stability of BIPIS. The scale alpha coefficients range among both sexes was $\alpha>0.90$. The value of Cronbach's alpha coefficients was similar to the results of the original study of BIPIS conducted by Callaghan et al [18]. But in the present study, alpha for all sub-scales was higher than 0.90 . Therefore, the findings of reliability measurement of the present study are in line with the original version. The result of retest coefficients in the present study was 0.62 , which was statistically significant $(\mathrm{P}<0.01)$ and also showed good reliability over time. The test-retest coefficients obtained was consistent with the study carried out by Callaghan et al. [18]. This indicator reflects the stability of the questionnaire over time.

The results of validity confirmation for BIPIS showed that its scale correlation coefficients are significant with all four

Table 1. Descriptive and demographic characteristics of participants

\begin{tabular}{ccccccc}
\hline \multirow{2}{*}{ Group } & Number & Percentage & \multicolumn{2}{c}{ Age } & \multicolumn{2}{c}{ Marital Status } \\
\cline { 4 - 7 } & & & Mean & SD & Male Frequency (\%) & Female Frequency (\%) \\
\hline Girl & 167 & 50.7 & 19.67 & $(1.64)$ & $133(79.6 \%)$ & $34(20.4 \%)$ \\
Boy & 162 & 49.3 & 20.84 & $(1.54)$ & $151(93.2 \%)$ & $11(6.8 \%)$ \\
Total & 329 & & 18.74 & $(2.84)$ & $284(86.4 \%)$ & $45(13.6 \%)$ \\
\hline
\end{tabular}

Table 2. The mean, standard deviation, Alpha coefficients and retest of Body Image Psychological Inflexibility Scale

\begin{tabular}{cccccccc}
\hline Sub-Scale & Material & Mean/ SD & \multicolumn{2}{c}{ Alpha Coefficient } & Retest Coefficient (N) & Split Up Coefficent \\
\hline BIPIS & $16-1$ & $56.3(12.2)$ & 0.93 & 0.90 & 0.92 & $0.62^{* *}$ & $0.87^{* *}$ \\
\hline$": \mathrm{P}<0.01$ & & & & & & & \\
\hline
\end{tabular}




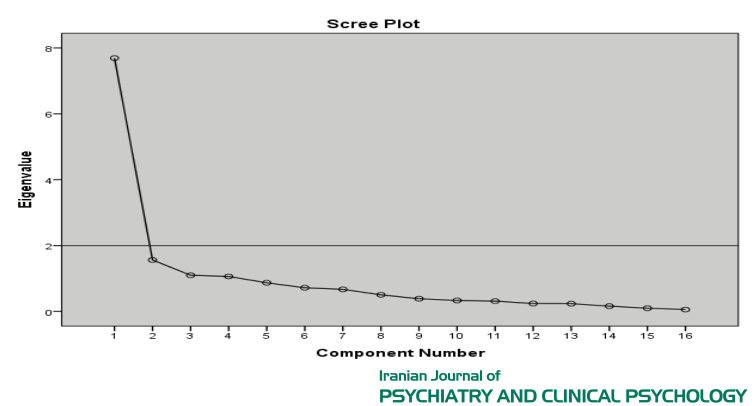

Figure 1. The slope of the graph (scree plot)

sub-scales of Meta-Cognitive control strategies, objectification of the thoughts, positive and negative Meta-Cognitive beliefs, and safety behaviors $(\mathrm{P}<0.01)$. In particular, all correlation coefficients between BIPIS and Meta-Cognition of physical deformities can theoretically confirm that BIPIS is a good self-reporting tool for measuring body image psychological inflexibility. The significance of this relationship suggests desirable relations between components. The results of correlation coefficients between sub-scales of BIPIS with Body Image Psychological Inflexibility Questionnaire [15] showed that there was a negative correlation between these two measures, which represented the desirable validity of BIPIS.

Assessment of reliability, validity and confirmatory factor analysis showed that BIPIS possessed adequate psychometric properties; the findings of this study also showed consistency with that of the original study [18]. The Persian version of BIPIS showed that it is a valid tool for assessing the psychological dimensions regarding body image. The calculated indices to evaluate the fitting of the BIPIS model showed Standardized Root Mean Square Residual (SRMR), Root Mean Square Error of Approximation (RMSEA), (X2/ degre free) indicators as the most prestigious fitness indicators [3337] supporting a good fitness model. This finding is in line with the results of the original scale of BIPIS [18]. In general, the desirable psychometric properties of BIPIS have a wide range of uses, ranging from the measurement of various psychological dimensions of physical resources to utilization at the management levels.

\section{Acknowledgments}

This research did not receive any specific grant from funding agencies in the public, commercial, or not-forprofit sectors.

\section{Conflict of Interest}

The authors declared no conflicts of interest. 


\title{
ويثَٔىهاى روانسنجى در مقياس انعطافنايذيرى روانشناختى تصوير بدنى \\ "سهراب اميرى'، ابوالقاسم يعقوبى'
}

\author{
ا- إكتراى روانشئاسى، كروه روانشئاسى، انشكده ادبيات و علومانسانى، دانشكاه اروميه، اروميه، ايران.

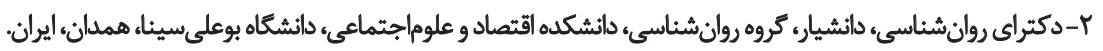

\begin{abstract}
حكيد

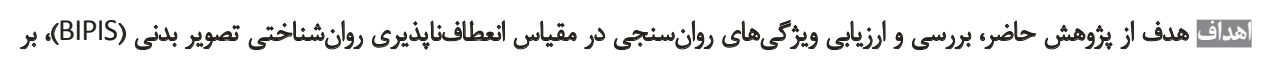

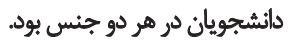

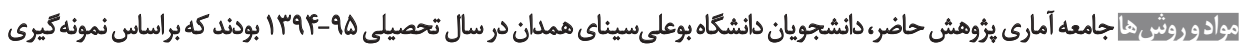

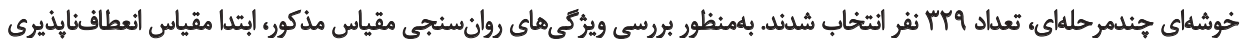

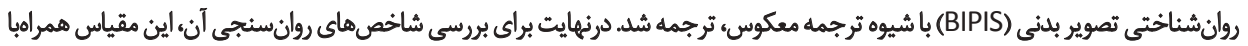

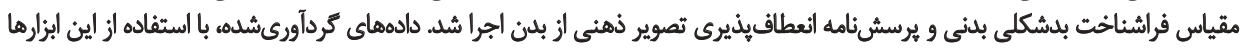

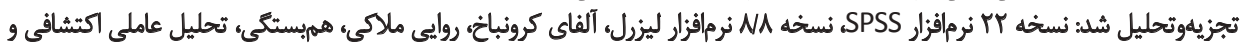
تأيبدى

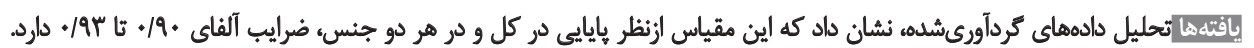

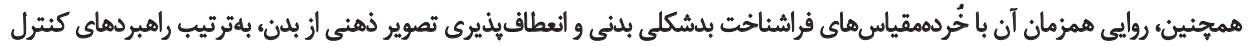

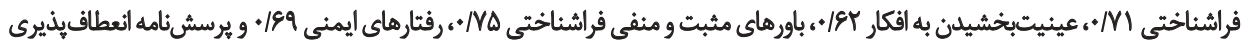
تصوير ذهنى ازٔ بدن (BI-AAQ)،

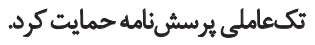

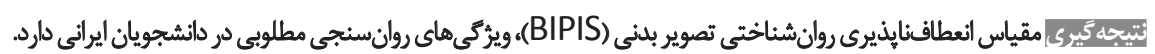

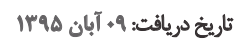

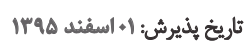

: Lotglouts

انعطافنايذيرى

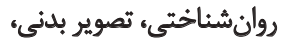
بلشكلي بليني، تحليل تصوير بلىني

اختلال بدريختانكارى بدنى، به اختلالات روانيزشكى همبوده،

مetar

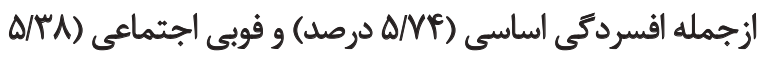

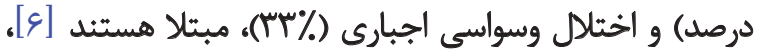

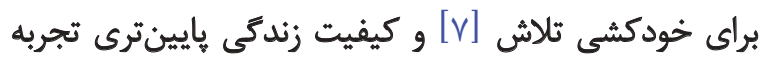

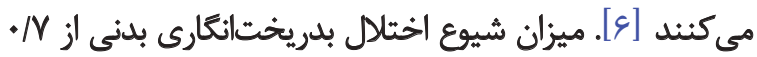

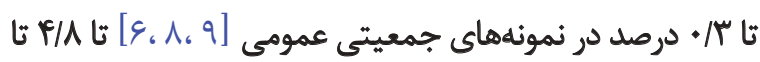

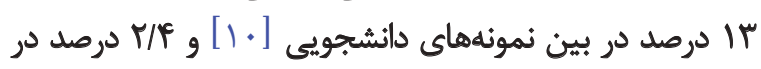

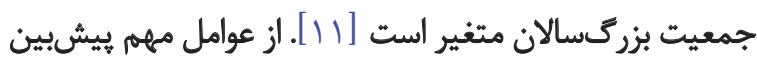

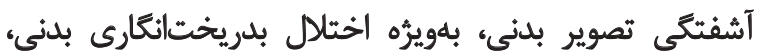

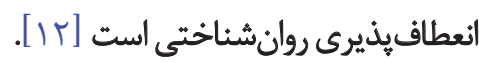

انعطافيذيرى روانشناختى عبارت است ازئ توانايى فرد براى

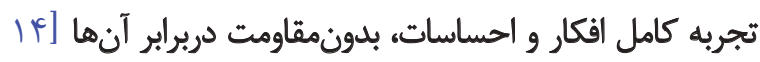

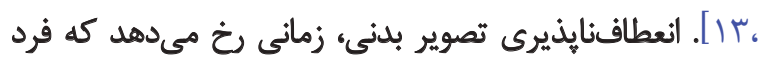

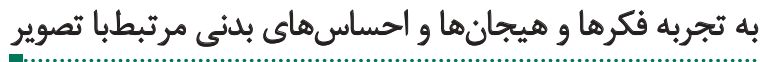
تصوير بدني، الكوهاى بيهيجيدهاي از نتخرشها، فكرها، هيجانها

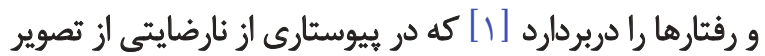

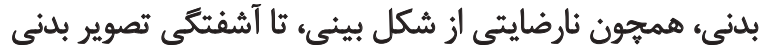

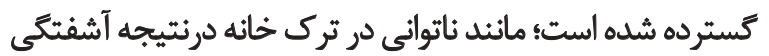

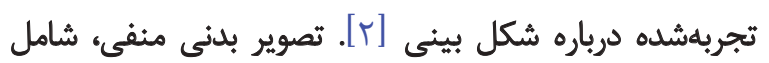

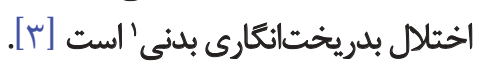

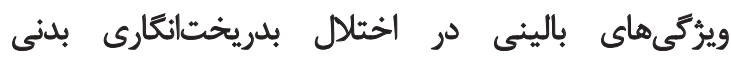

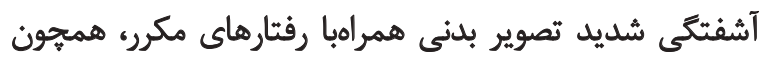

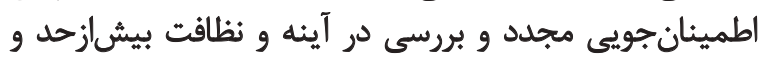

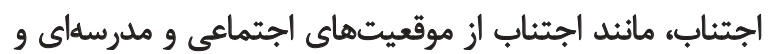

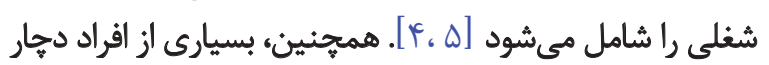

1. Body Dysmorphic Disorder (BDD)

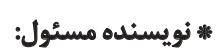

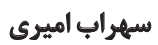

نشانىى: اروميه، دانشعاه اروميه، دانشكده ادبيات و علوم|نسانيء كروه روانشناسى.

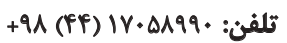
يست الكترونيكي: بm:arirysohrab@yahoo.com 
بدن تمركز دارد. اين در حالى است كه نشائهشناسي اختلال

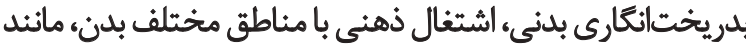

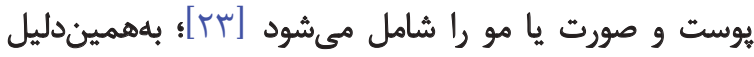

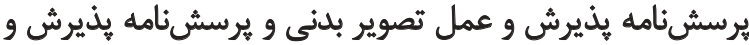

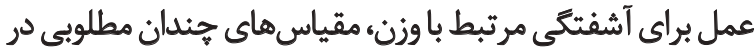

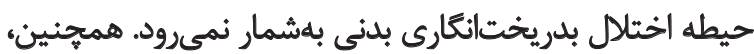

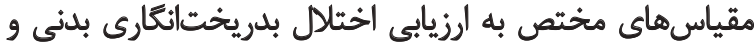

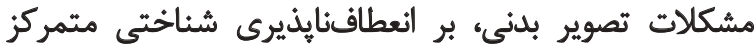

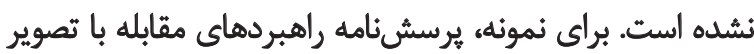

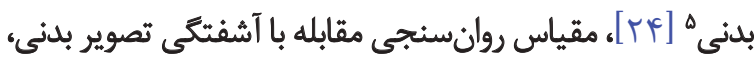

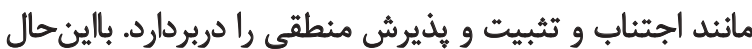

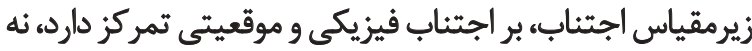

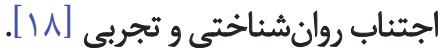
درمجموع باتوجهبه آنجه بيان شد، تصوير بدنى بهعنوان عامل مهرم

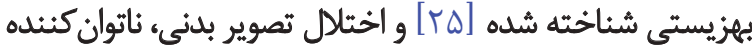

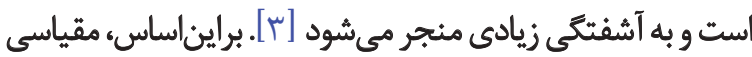

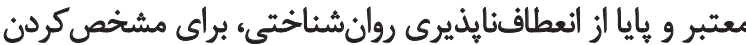

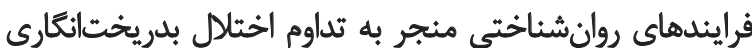

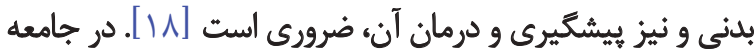

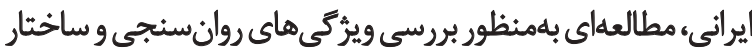

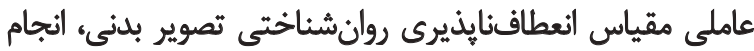

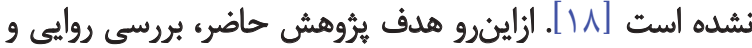

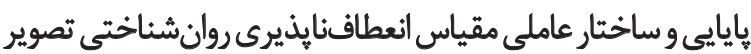
بدنى، در نمونه جمعيت دانشجويى ايرائى است.

شيوه يروهش حاضر، توصيفىروانسنجى بود و جامعه آمارى

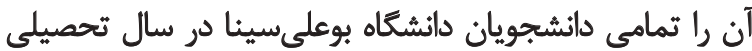

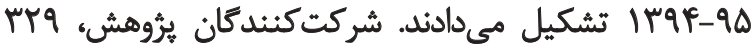

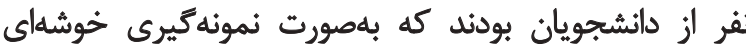

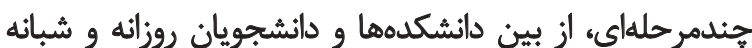

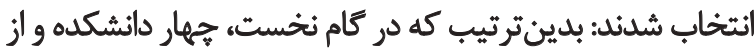

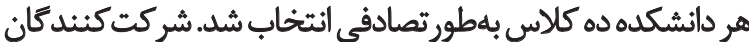

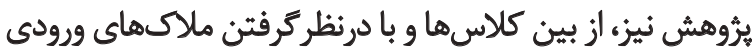

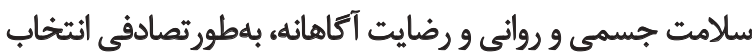

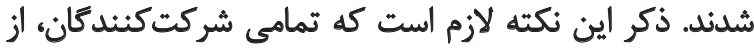

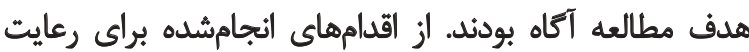

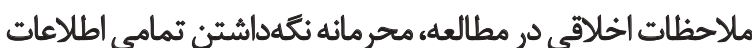

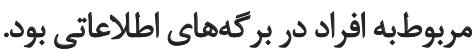
براى جمعآورى دادهها،درابتدايثروهشكَردربين شركت كنيندان

\footnotetext{
5. Body Image Psychological InfleXibility Scale (BIPIS)
}

بدنى تمايلى ندارد و تلاش مي كند ثا شكل يا ميزان وقوع اين

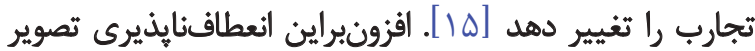

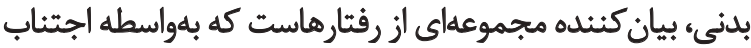

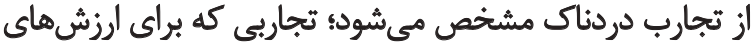

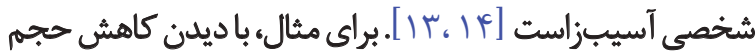

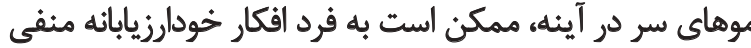
دست دهد كه به آشفتتى و احساس درماندگى فرد منجر شود. اللوى انعطافنايذيرى روانشناختى، بيان مى كند كه اين فرد

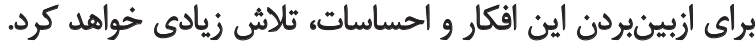

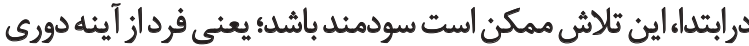

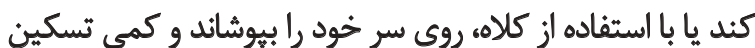

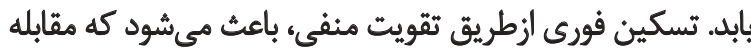

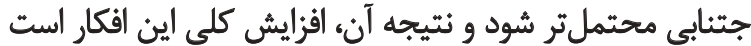

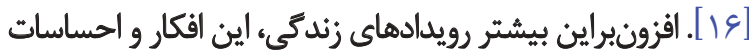

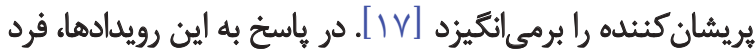

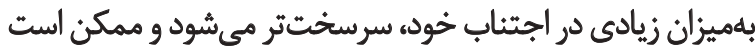

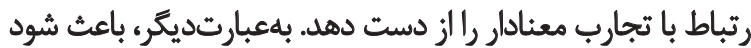

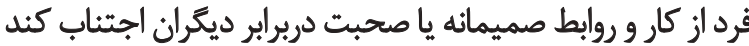

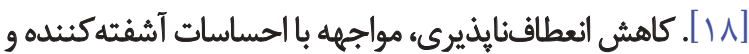

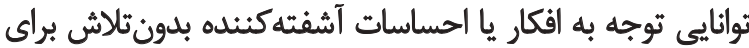

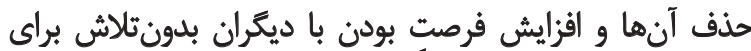

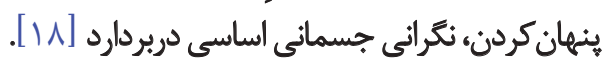

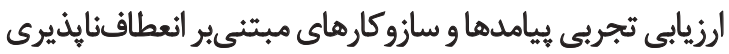

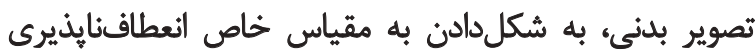

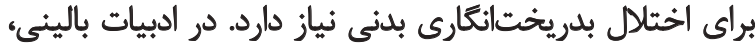

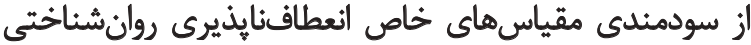

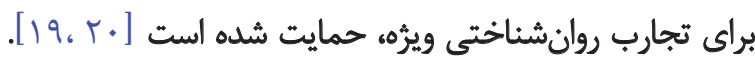

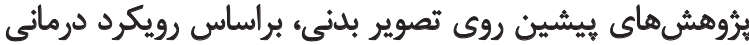

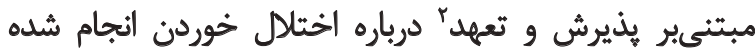

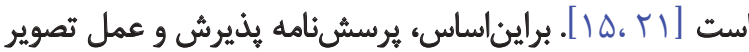

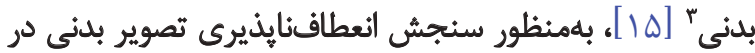

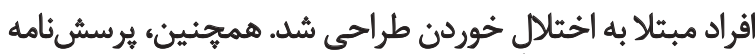

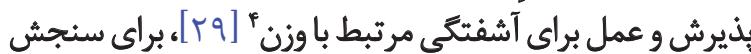

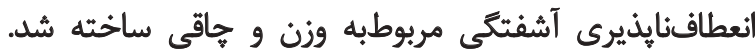

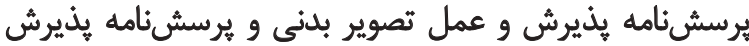

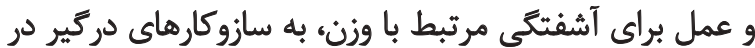

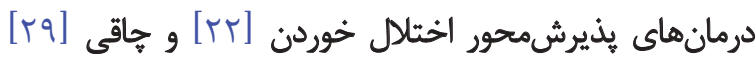

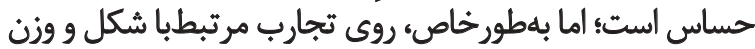

2. Acceptance and Commitment Therapy (ACT)

3. Body Image-Acceptance and Action Questionnaire (BI-AAQ) 4. Acceptance and Action Questionnaire for Weight Related Distress (AAQ-W) 


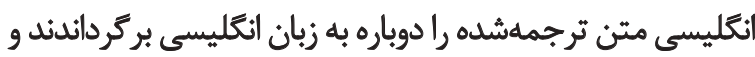

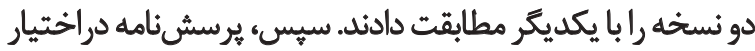

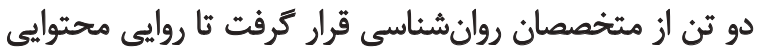

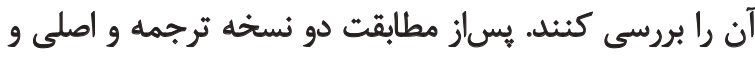

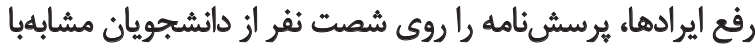

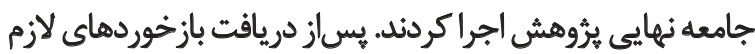

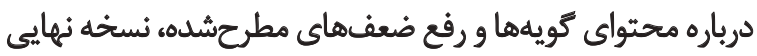
يرسش نامه را براي استفاده تهيه كردند.

\section{يرسش نامه فراشناخت بدشكلى بدنى "}

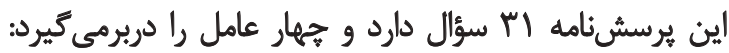

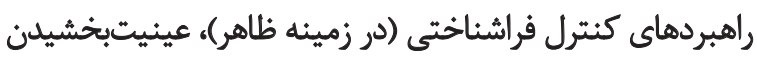

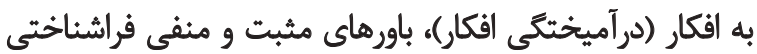

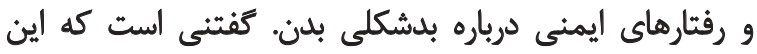

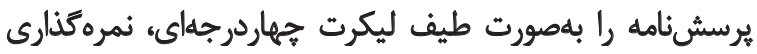

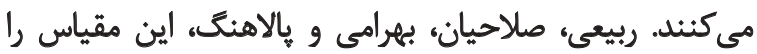

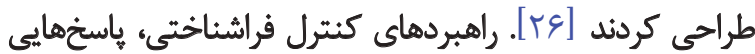

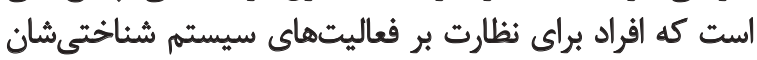

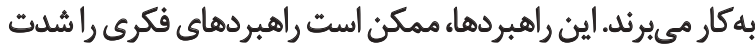

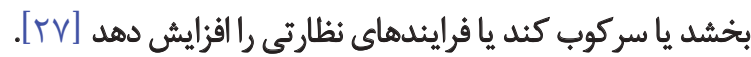

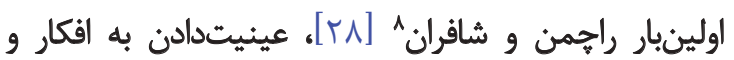

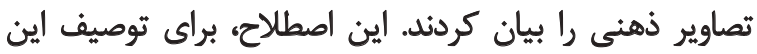

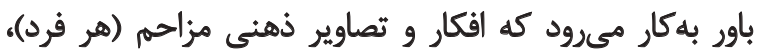

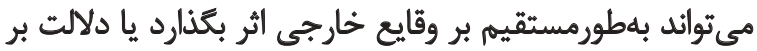

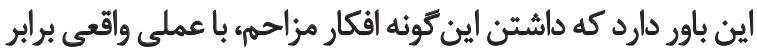

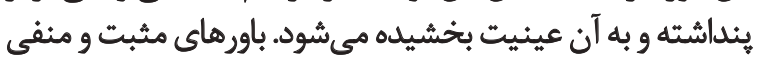

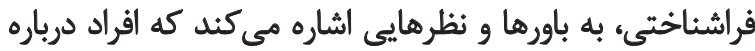

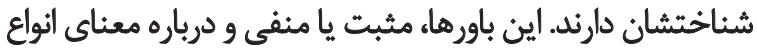

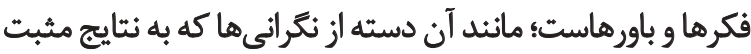

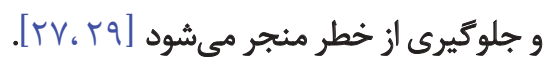

رفتارهاى ايمنى نيز، هاسخهايى است كه باعث حفظ

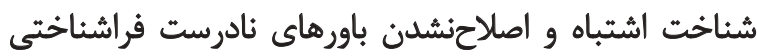

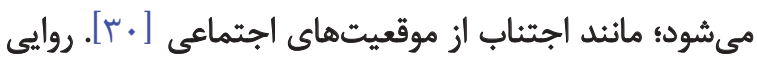

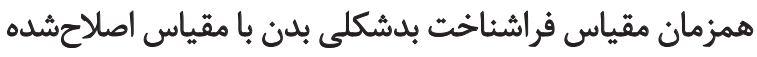

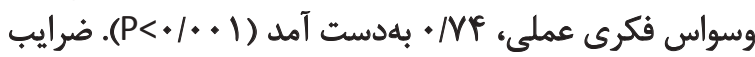

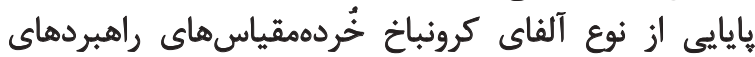

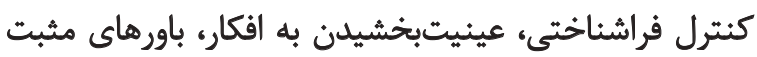

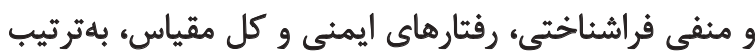

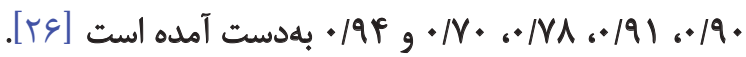

7. Body Dysmorphic Metacognition Questionnaire (BDMQ) 8. Rachman \& Shafran
يُروهش حضور يافت و فرايند ياسخدهى را توضيح داد. سيس،

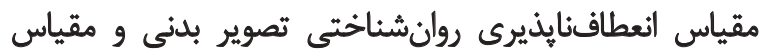

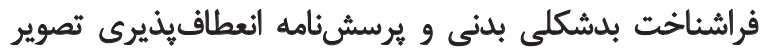

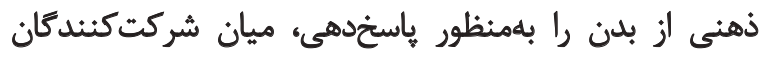

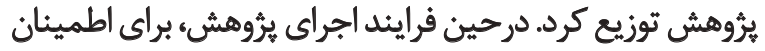

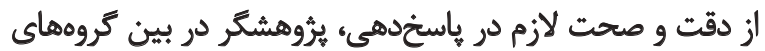

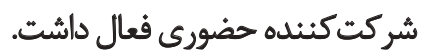

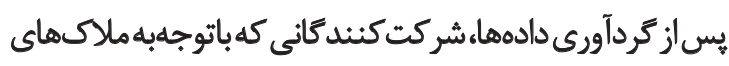

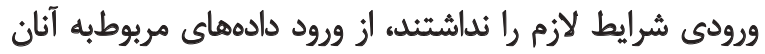

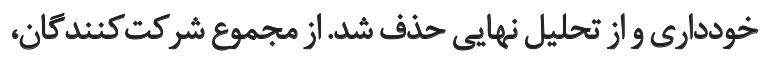

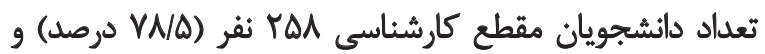

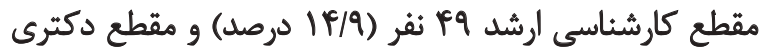

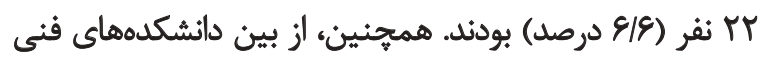

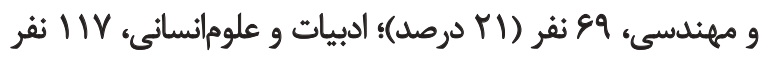

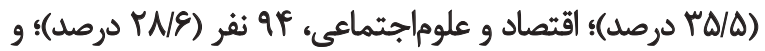

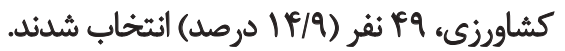

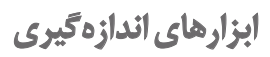

\section{مقياس انعطافنايذيرى روانشناختى تصوير بدنى}

مقياس انعطافنايذيرى روانشناختى تصوير بدنى، شانزده ماده

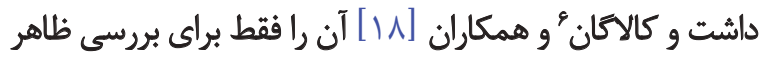

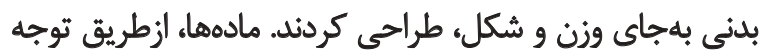

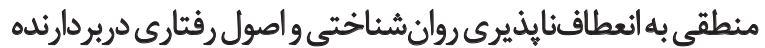

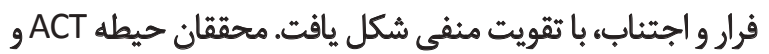

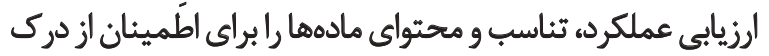

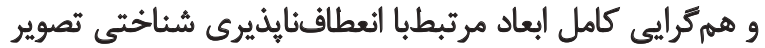
بدنى در اختلال بدريختانكارى بدنى بازنكرى كردند.

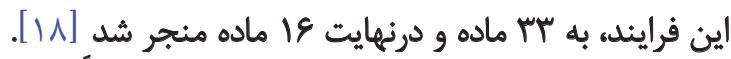

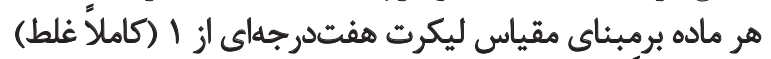

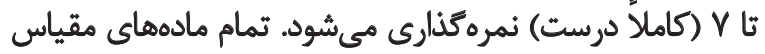

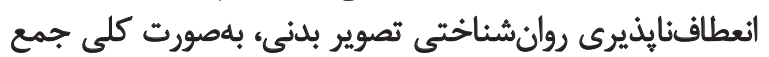

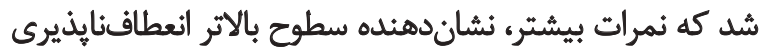

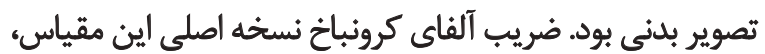

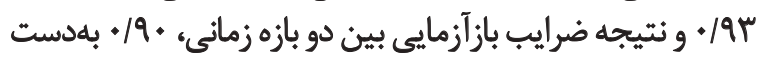

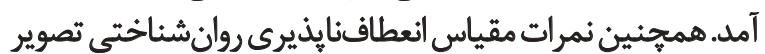

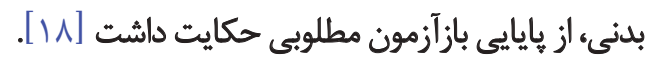
بلمنظور تهيه نسخه فارسى مقياس انعطافنإيذيرى

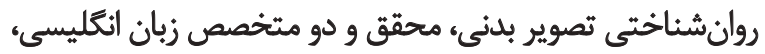

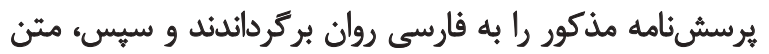

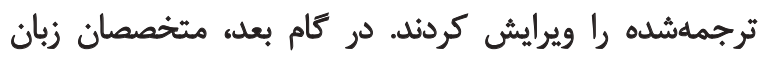

6. Callaghan 


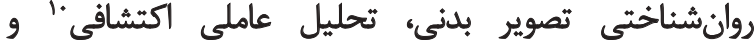

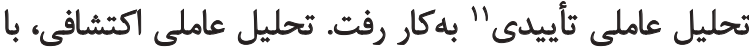

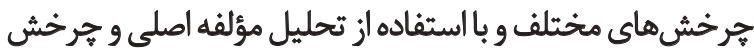

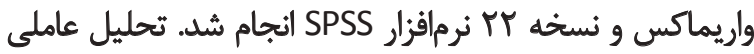

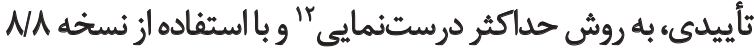

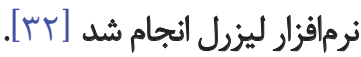

ياقتثهها

در جدول شماره ا، ويرَّى هاى توصيفى و جمعيتشناختى شركت كنندكان ارائه شده است.

بإيائي

براى بررسى ثبات مقياس انعطافنايذيرى روانشناختى

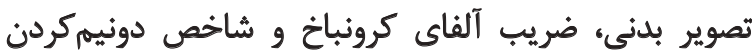

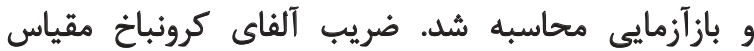

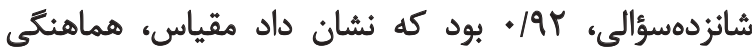

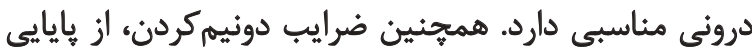

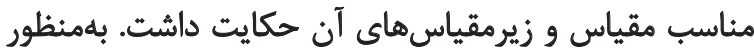

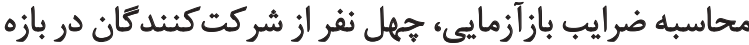

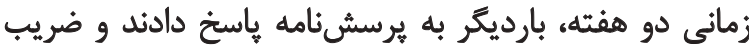

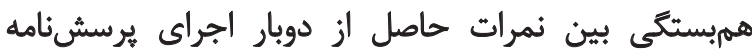

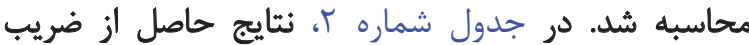

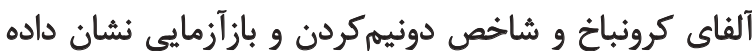

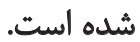

نتايج جدول شماره r، نشان ميدهد كه ضرايب آلفاي كرونباخ،

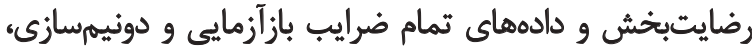

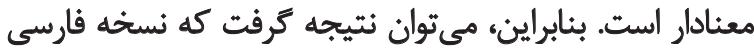

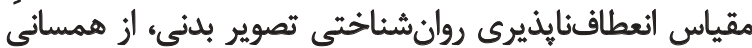
درونى مطلوبى برخوردار است.

10. EXploratory factor analysis

11. Confirmatory factor analysis

12. MaXimum likelihood method

\section{يرسش نامه انعطاف يذيرى تصوير بدن"}

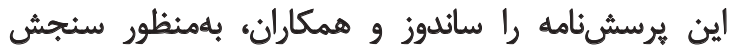

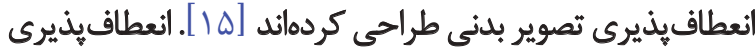

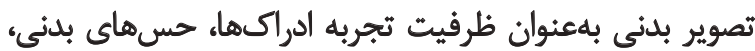

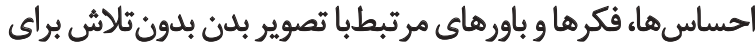

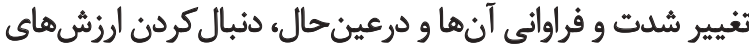

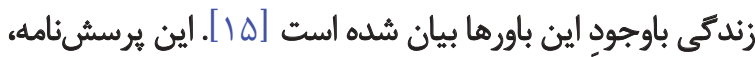

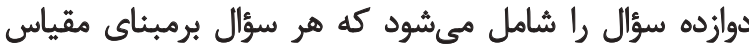

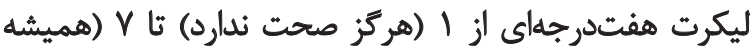

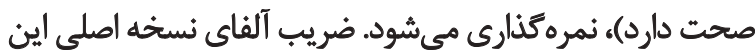

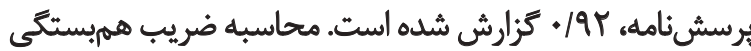

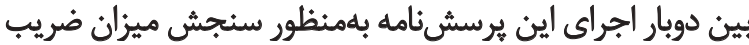

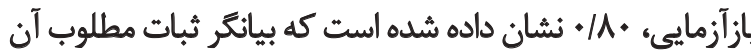

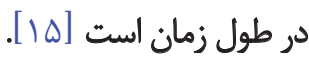

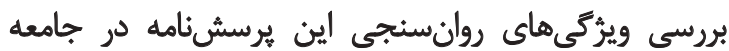

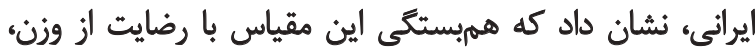

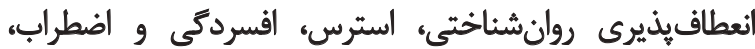

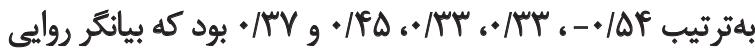

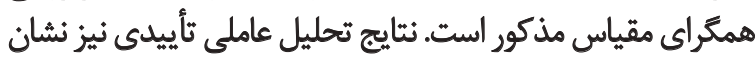

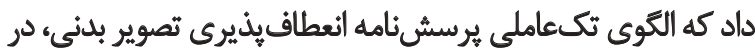

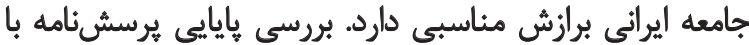

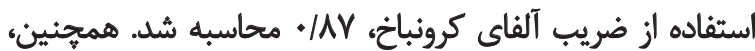

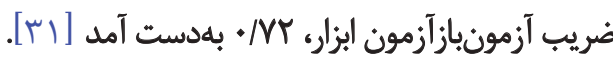

$$
\text { روش ثجزيلورقحليل دادهها }
$$

براى بررسى ثبات مقياس انعطافنايذيرى روانشناختى تصوير

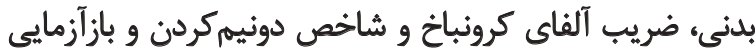

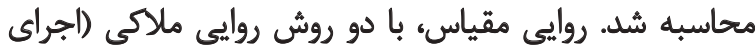

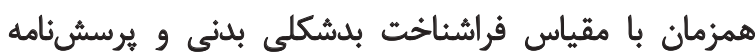

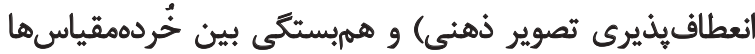

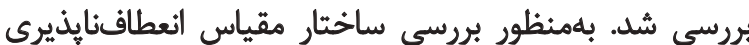

9. Body Image FleXibility (BDF)

\begin{tabular}{|c|c|c|c|c|c|}
\hline \multicolumn{2}{|c|}{ وضعيت تأهل } & \multirow{2}{*}{ (ميانكين/انحراق } & \multirow{2}{*}{ درصد } & \multirow{2}{*}{ تعداد } & \multirow{2}{*}{ كروه } \\
\hline مثأهل ( ميانكين/انحراف استاندارد) & مجرد (ميانكين/انحراف استاندارد) & & & & \\
\hline$M e(r-/ \varphi)$ & imp(Na/s) & IV/EV(V/EP) & $\Delta \cdot / N$ & IEN & دختر \\
\hline $11(\varepsilon / 1)$ & $\mid 01 / 91 / T)$ & $r \cdot / A T(Y / \Delta F)$ & $r q / r$ & Iar & بـسر \\
\hline$P \Delta(\mid Y / \theta)$ & $\operatorname{rAP}(\Lambda \in \mathbb{N})$ & $W M^{e}(Y / A P)$ & & $M q$ & كل \\
\hline
\end{tabular}

جدول ا. ويرُّى هاي توصيفى و جمعيتشناختى شركت كنيندان برُوهش 
جدول r. ميانكين، أنحراف استاندار، ضرايب آلفا و بازآزمايى مقياس ائعطافئايذيرى تصوير بلدى

\begin{tabular}{|c|c|c|c|c|c|c|c|}
\hline \multirow{2}{*}{ ضريب دونيه كردن } & \multirow{2}{*}{ ضريب بازآزهايي (*) } & \multicolumn{3}{|c|}{ ضريب آلفا } & \multirow{2}{*}{ ميانغين /انيحراف اسيتاندارد } & \multirow{2}{*}{ هواد } & \multirow{2}{*}{ مُردهمقياس } \\
\hline & & كل & يسير & هختر & & & \\
\hline$*$ * /AV & * ar &.$/ 94$ & .19 &.$/ 95$ & $\Delta \& / T(I T / N)$ & 1851 & BIPIS \\
\hline
\end{tabular}

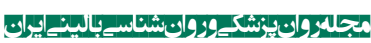

${ }^{* *} \mathrm{P}<.1 .1$

تهليل عاملي

روائي

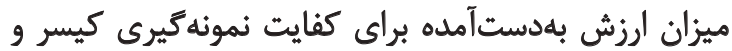

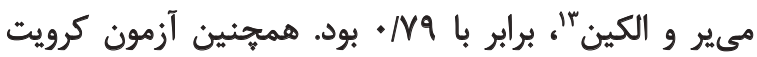

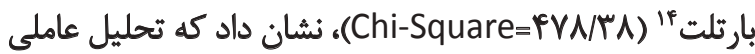
امكانئير است. درادامه و در جدول شماره باء ميزان اشتراك

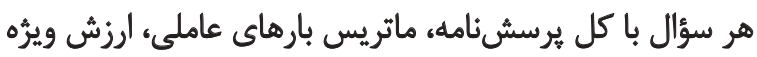

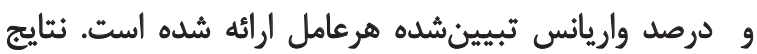

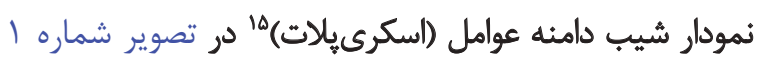

13. Kaiser-Meyer-Olkin Measure of Sampling Adequacy (KMO) 14. Bartlett's test of sphericity 15. Scree plot
ضرايب همربستگى بين مقياس انعطافنايذيرى روانشناختى تصوير

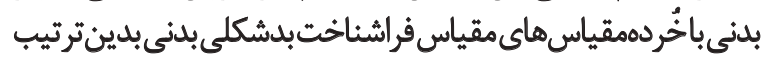

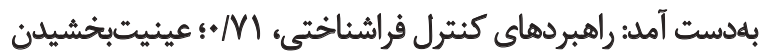

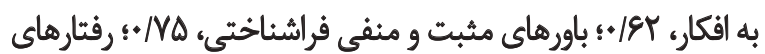

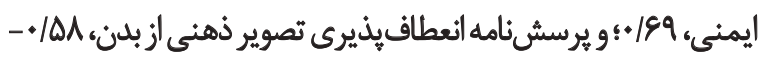

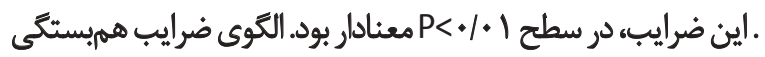

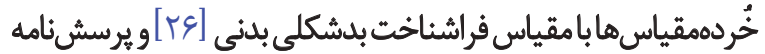

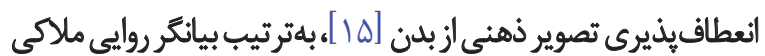
همزمان و نيز روايي واكرايي مطلوب نسخه فارسي انعطافنايذيري زئني

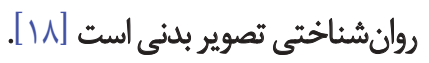

جدول ب. ميزان اشتراك هر سؤال با برسشنامه و ارزش ويرُه و درصد تبيين واريانس هرعامل هيساز جرخش واريماكس براساس تحليل مؤلفههاى اصلى

\begin{tabular}{|c|c|c|c|}
\hline 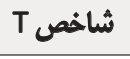 & بار عاملى & 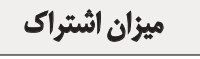 & مواد \\
\hline $4 / 4 q$ & $+\mid \Delta \&$ & 189 & 1. خيلى مراقب ظاهرم هسته. \\
\hline$f / M$ & $.18 \mathrm{~V}$ & . $\mathrm{NA}$ & r. وقتى احساس بدى درباره ظاهرم داشته باشم، مأيوس مى شوم. \\
\hline r/Mr & .18. & . No & كا. ييش ازاينكه كامهاى ههمى در زندكى بردارم، افكار و احساساتم درباره ظاهرم بايد تغيير كند. \\
\hline$r / M V$ & $\cdot / \Delta \cdot \Delta$ & $\cdot / \Delta \Delta$ & F. زمان زيادى صرف فكركردن درباره اين موضوع مىكنم كه درنظر ديكران وضعيت ظاهرم جكونه بهنظر مىآيد. \\
\hline $9 / 9$ & $\cdot|A F|$ & - $/$ Ar & هـ اكر بتوائهم افكار منفى ام درباره ظاهر ر را كثترل كثم، كثترل بهترى روى زندكى ام خواهم داشت. \\
\hline $9 / . r$ & . Ner & - Na & و براى داشتن زئلى بهتر، نياز دارم تا ظاهرم راكتترل كنه. \\
\hline$\Delta / \%$ & $\cdot M$ & .180 & V. وقتى درباره اين موضوع فكر هى كنم كه درنظر ديكران هكونه ديله مى شوم، انجام كار ديكر، برايم دشوار الست. \\
\hline $\mathrm{V} / \mathrm{M}$ & - /AAr & $\cdot M$ & ه اكر ظاهرم مرا آزار ثلهد، روابط من بهتر خواهد بود. \\
\hline$\varphi / Q 1$ & .189 & $\cdot M$ & 9. متوجه هستم كه ظاهرم نواقصى دارد و من آنها را ميذيرم. \\
\hline$r / g r$ & $\cdot / \Delta D Q$ & / $/ \Delta r$ & • ا. خودم را بهواسطه ديدكاههاى ديكران درباره ظاهرم مى شناسه. \\
\hline$\Delta / a r$ &.$/ \mathrm{MAq}$ &.$/ \mathrm{N}$ & 11. خودارزيابى و احساس منفى درباره ظاهرم موجب مشكلاتى در زئدكىام ميشود. \\
\hline$\Delta / \cdots$ & $\cdot M I F$ & $\cdot|A|$ & r I. افكار منفى من درباره ظاهرم، باعث مي شود از زندكى لذت نبرم. \\
\hline P/r & - Iepp & $\cdot / M 9$ & با. اكر قسمتى از ظاهر بدنىام را دوست نداشئه باششم، خودم رانيز دوست ندارم. \\
\hline $9 / 1 f$ & - mar & $.18 \mathrm{~A}$ & با. آكر بتوانم تغييراثى در ظاهرم ايجاد كنم، آن وقت شادتر خواهم بود. \\
\hline$\Delta / V \Delta$ & - NaP &.$/ 84$ & 1ه ا. خلقم از ظاهرم بسيار ثأثير مي يذيرد. \\
\hline$\varphi / \&$ & $.18 \mathrm{r}$ & س & ع. مى خواهم يس اندازهاى زندكى ام را صرف اصلاح نواقص ظاهرىام كنه. \\
\hline \multicolumn{2}{|c|}{ st/g9 } & درصد ثبيين واريانس & الرزش ويرهه \\
\hline
\end{tabular}




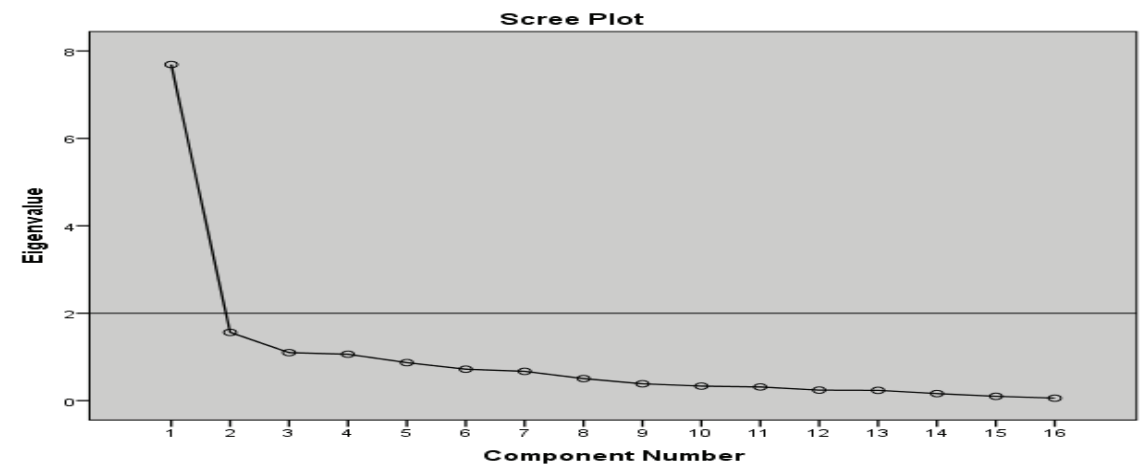

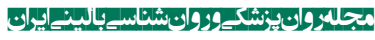

تصوير ا. نمودار شيب دامنه عوامل (اسكرى يلات)

ايجاز، مانند ريشه خطاى ميانكين مجذورات تقريب (RMSEA)؛

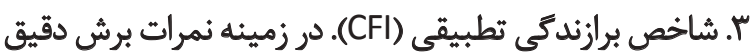

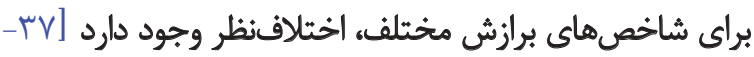

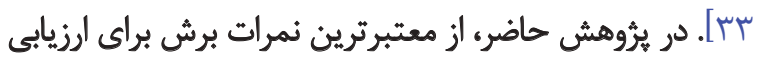

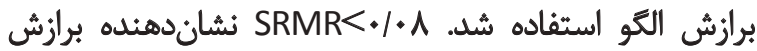

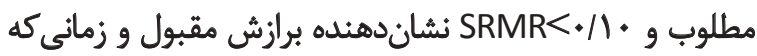

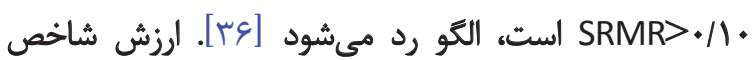

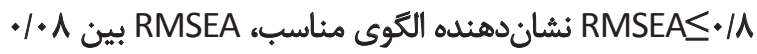

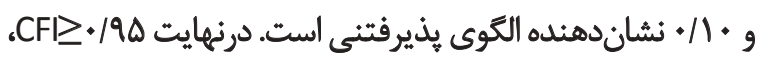
نشاندهنده برازش مناسب الكوست [عب].

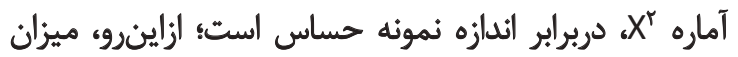

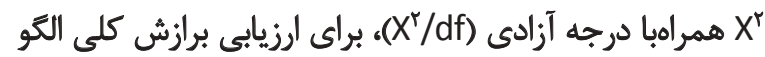

و تحليل مؤلفههاى اصلى، وجود يك مؤلفه با ارزشهاى بيشتر

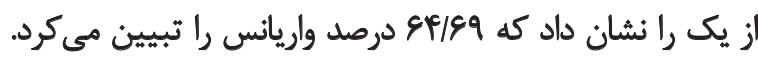

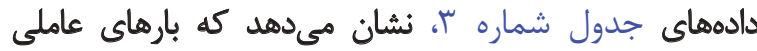

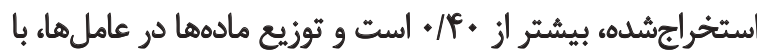

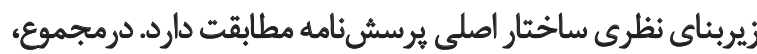

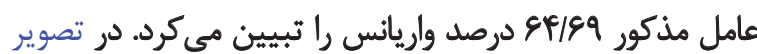

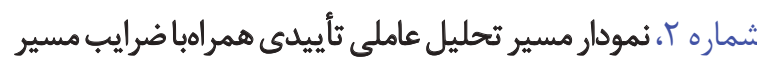
و همجنين در جدول شماره أ، شاخص تى ملاحظه مى شمود.

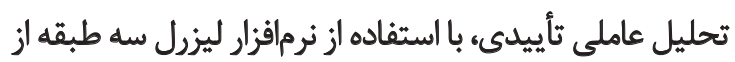

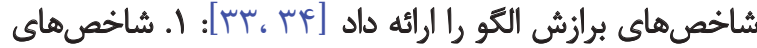

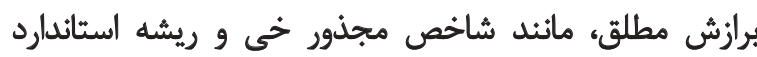

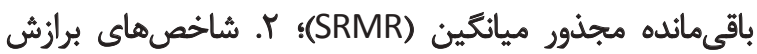

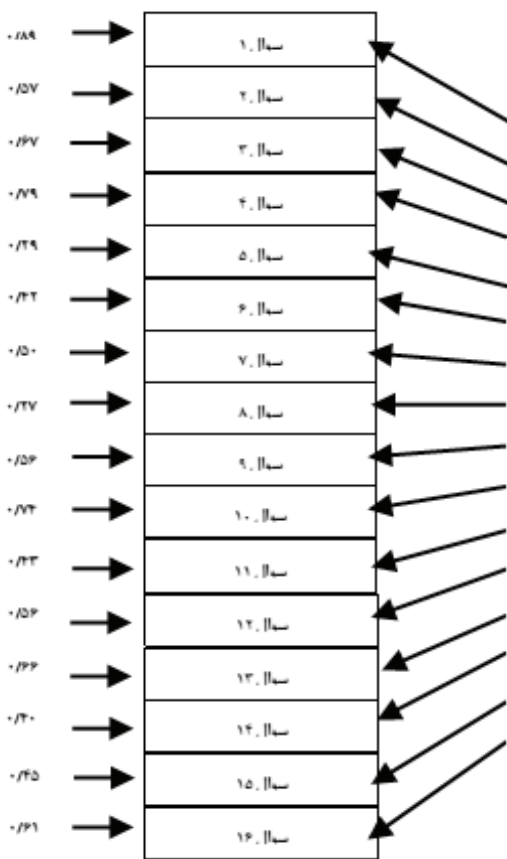

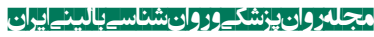

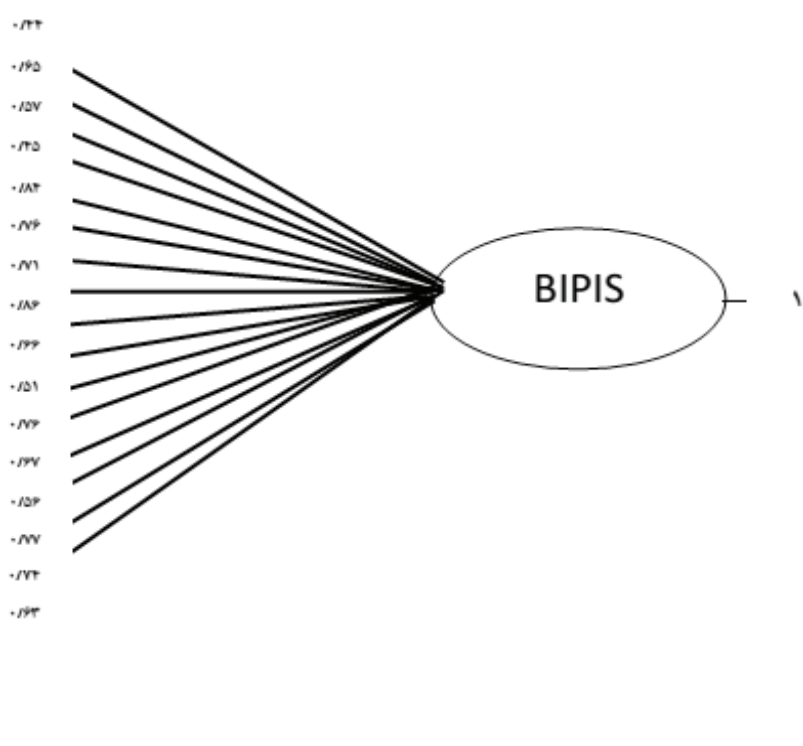

تصوير آ نمودار تحليل عاملي ثأييدى و ضرايب مسير انعطافنايذيرى روانشئاختي تصوير بلنى 
جدول P. جاخصهاى برازش الكو

\begin{tabular}{|c|c|c|c|c|c|c|c|c|c|c|c|}
\hline $\mathrm{AGFI}^{8}$ & $\mathrm{GFI}^{7}$ & $\mathbf{I F |}^{6}$ & $\mathrm{CFI}^{5}$ & $\mathbf{N N F I}^{4}$ & $\mathbf{N F I}^{3}$ & SRMR $^{2}$ & RMSEA $^{1}$ & $\mathbf{P}$ & df & $\mathrm{X}^{2}$ & شاخص \\
\hline . /ar & $\cdot \mid M$ & .19 &.$/ 91$ & $\cdot M$ &.$/ 97$ & 1.4 & .1 .1 & $>.1 .0$ & 1.4 & IVI/aY & ارزش \\
\hline
\end{tabular}

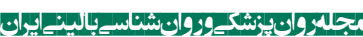

1. Root Mean Square Error of Approximation

2. Standardized Root Mean Square Residual

3. Normed Fit Index

4. Non-Normed Fit Index

بدنى، نشان داد كه ضرايب همبستّى مقياس انعطافنايذيرى

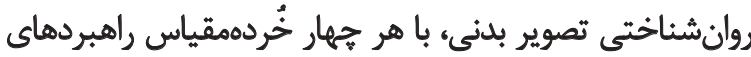

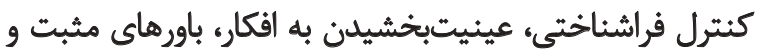

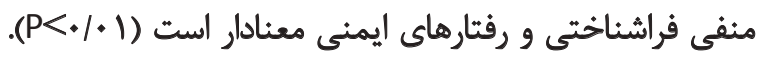

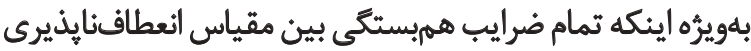

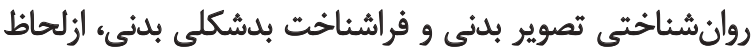

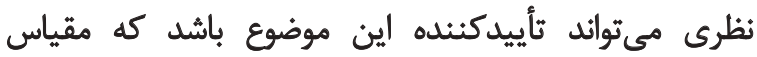

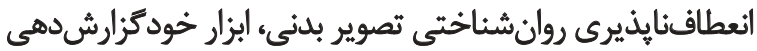

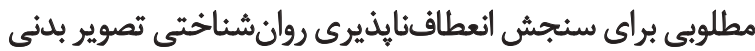

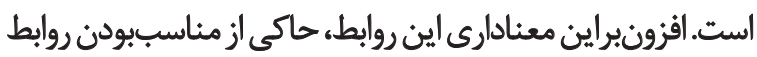

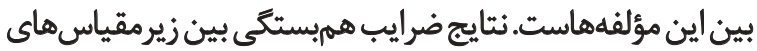

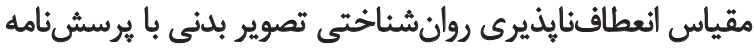

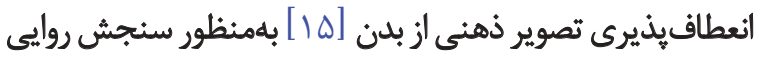

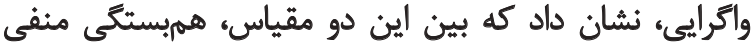

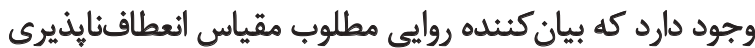
روانشناختى تصوير بدنى است

تحليل هاى يايايى و روايى و تحليل عاملى تأييدى، نشاندهئدي

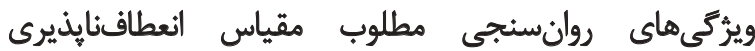

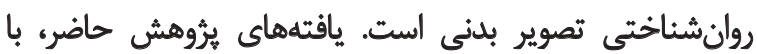

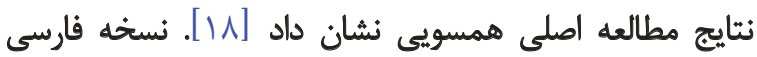

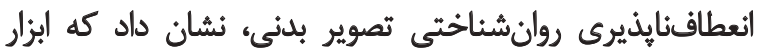

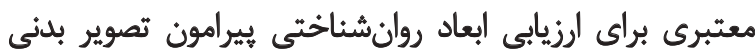

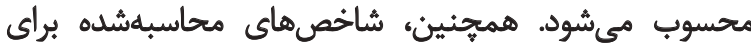

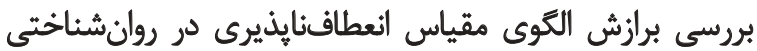

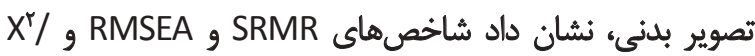

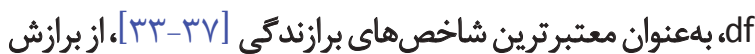

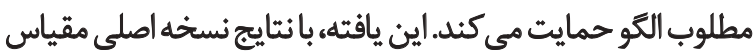

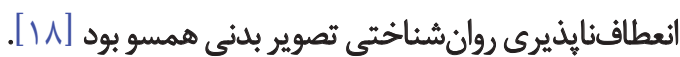

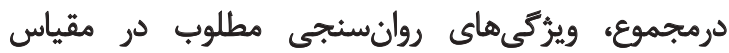

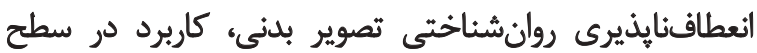

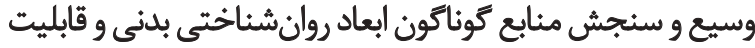

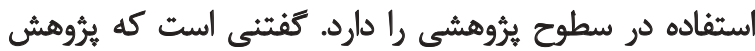

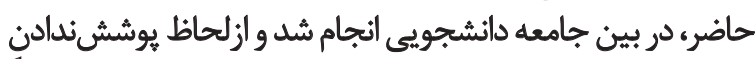

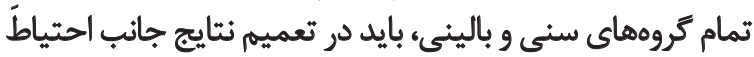

5. Comparative Fit Index

6. Incremental Fit Index

7. Goodness of Fit Index

8. Adjusted Goodness of Fit Index

محاسبه شد. ميزان XYld

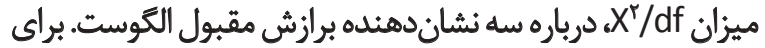

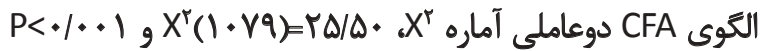

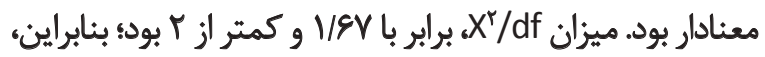

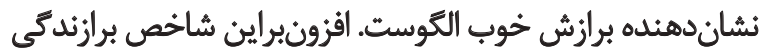

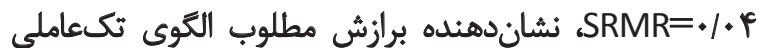

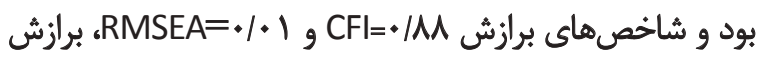

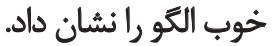

ث

هدف برثوهش حاضر، بررسى ويرّكى هائ روانسنجى در مقياس

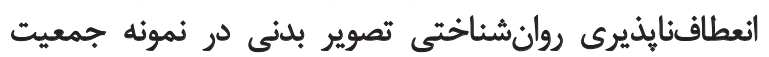

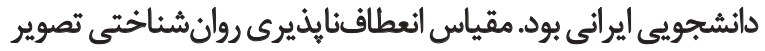

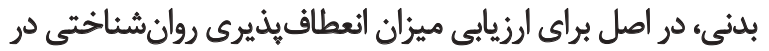

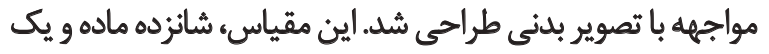

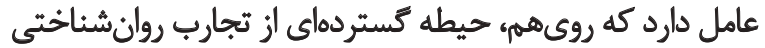

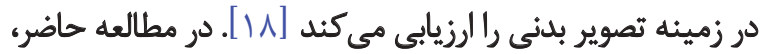

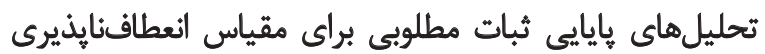

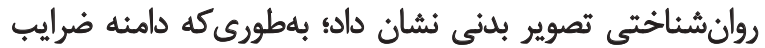

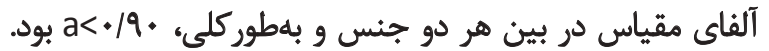

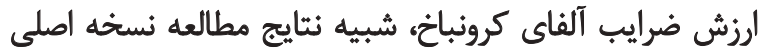

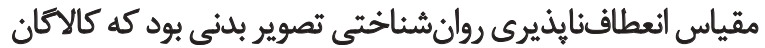

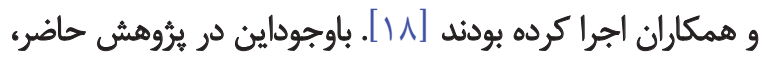

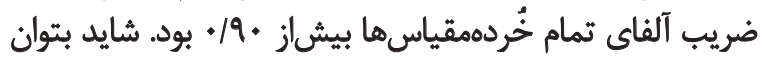

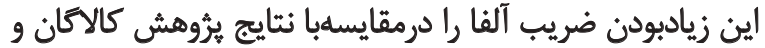

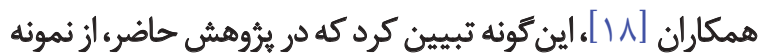

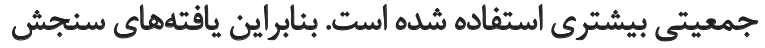

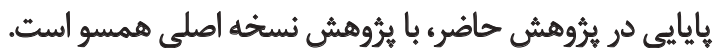
در برؤهش حاضر، نتايج ضرايب بازآزمون، كاع| • و معنادار بود

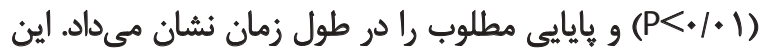

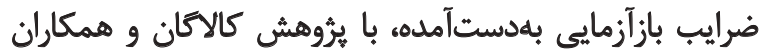

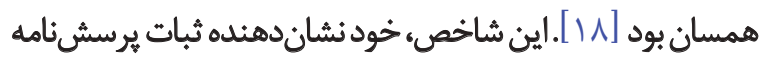

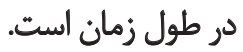

نتايج بررسى روايى مقياس انعطافنايذيرى روانشناختى تصوير 


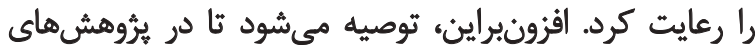

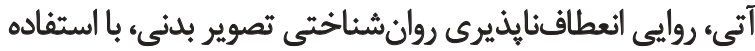

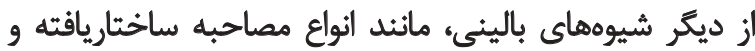

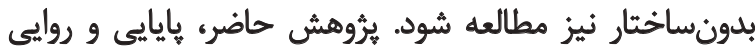

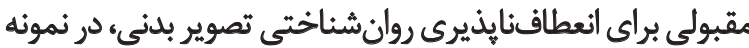

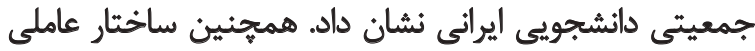

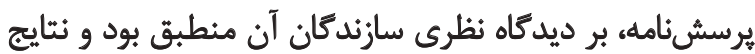

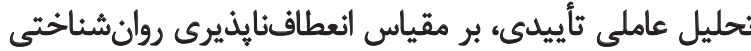

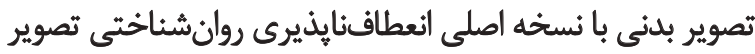

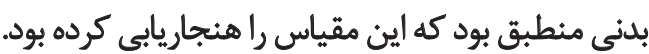

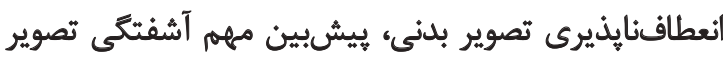

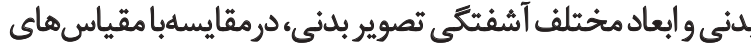

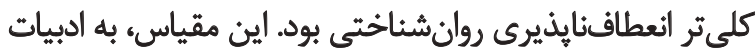

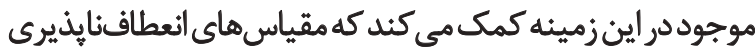
روانشناختى خاص يك موقعيت، ممكن است بردين براي اشكال

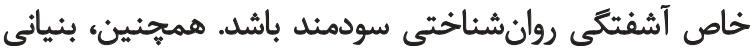

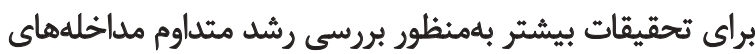

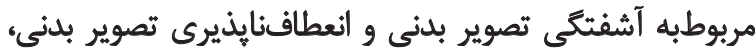

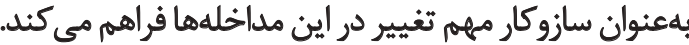

$$
\text { سياسكَّزارى }
$$

بدينوسيله از تمامي دانشجويان دانشكاه بوعلى بـينا قدرداني

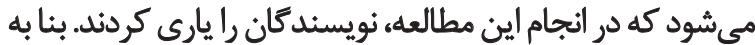

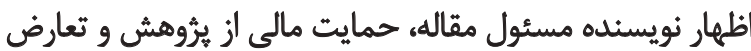

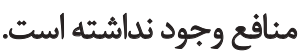




\section{References}

[1] Cash T, Smolak L. Body image. New York: Guilford Press; 2011.

[2] Cash T, Phillips K, Santos M, Hrabosky J. Measuring negative body image: Validation of the body image disturbance questionnaire in a nonclinical population. Body Image. 2004; 1(4):363-372. doi: 10.1016/j.bodyim.2004.10.001

[3] Greenberg JL, Delinsky SS, Reese HE, Buhlmann U, Wilhelm S. Body image. In: Grant J, Potenza M, editors. Young Adult Mental Health. New York: Oxford University Press; 2010.

[4] American Psychiatric Association. Diagnostic \& statistical manual of mental disorders. $5^{\text {th }}$ ed. Washington, D.C.: American Psychiatric Association; 2013.

[5] Phillips K, Didie E, Menard W, Pagano M, Fay C, Weisberg R. Clinical features of body dysmorphic disorder in adolescents and adults. Psychiatric Research. 2006; 141(3):305-314. doi: 10.1016/j. psychres.2005.09.014

[6] Phillips K, Menard W, Fay C, Weisberg R. Demographic characteristics, phenomenology, comorbidity, and family history in 200 individuals with body dysmorphic disorder. Psychosomatics. 2005; 46(4):317-325. doi: 10.1176/appi.psy.46.4.317

[7] Veale D, Boocock A, Gournay K, Dryden W. Body dysmorphic disorder: A survey of fifty cases. British Journal of Psychiatry. 1996; 169(2):196-201. doi: 10.1192/bjp.169.2.196

[8] Buhlmann U, Glaesmer H, Mewes R, Fama JM, Wilhelm S, Brähler E, et al. Updates on the prevalence of body dysmorphic disorder: A population based survey. Psychiatry Research. 2010; 178(1):171-175. doi: 10.1016/j.psychres.2009.05.002

[9] Rief W, Buhlmann U, Wilhelm S, Borkenhagen A, Brhler E. The prevalence of body dysmorphic disorder: A population-based survey. Psychological Medicine. 2006; 36(6):877. doi: 10.1017/ s0033291706007264

[10] Bohne A, Wilhelm S, Keuthen N, Florin I, Baer L, Jenike M. Prevalence of body dysmorphic disorder in a German college student sample. Psychiatry Research. 2002; 109(1):101-104. doi: 10.1016/s0165-1781(01)00363-8

[11] Koran LM, Abujaoude E, Large MD, Serpe RT. The prevalence of body dysmorphic disorder in the United States adult population. CNS Spectrums. 2008; 13(4):316-322. doi: 10.1017/ s1092852900016436

[12] Callaghan G, Duenas J, Nadeau S, Darrow S, Van der Merwe J, Misko J. An empirical model of body image disturbance using behavioral principles found in functional analytic psychotherapy and acceptance and commitment therapy. International Journal of Behavioral Consultation and Therapy. 2012; 7(2-3):16-24. doi: 10.1037/h0100932

[13] Bond FW, Hayes SC, Baer RA, Carpenter KM, Guenole N, Orcutt HK, et al. Preliminary psychometric properties of the acceptance and action questionnaire II: A revised measure of psychological inflexibility and experiential avoidance. Behavior Therapy. 2011; 42(4):676-688. doi: 10.1016/j.beth.2011.03.007

[14] Hayes SC, Strosahl KD, Wilson KG. Acceptance and commitment therapy: The process and practice of mindful change. $2^{\text {nd }} \mathrm{ed}$. New York: Guilford Press; 2011.

[15] Sandoz E, Wilson K, Merwin R, Kate Kellum K. Assessment of body image flexibility: The Body Image-Acceptance and Action
Questionnaire. Journal of Contextual Behavioral Science. 2013; 2(1-2):39-48. doi: 10.1016/j.jcbs.2013.03.002

[16] Wenzlaff RM, Wegner DM. Thought suppression. Annual Review of Psychology. 2000; 51(1):59-91. doi: 10.1146/annurev. psych.51.1.59

[17] Hooper N, Saunders J, McHugh L. The derived generalization of thought suppression. Learning \& Behavior. 2010;38(2):160-168. doi: $10.3758 / \mathrm{lb} .38 .2 .160$

[18] Callaghan G, Sandoz E, Darrow S, Feeney T. The body image psychological inflexibility scale: Development and psychometric properties. Psychiatry Research. 2015; 226(1):45-52. doi: 10.1016/j. psychres.2014.11.039

[19] Gregg J, Callaghan G, Hayes S, Glenn-Lawson J. Improving diabetes self-management through acceptance, mindfulness, and values: A randomized controlled trial. Journal of Consulting and Clinical Psychology. 2007; 75(2):336-343. doi: 10.1037/0022006x.75.2.336

[20] Lillis J, Hayes S, Bunting K, Masuda A. Teaching acceptance and mindfulness to improve the lives of the obese: A preliminary test of a theoretical model. Annals of Behavioral Medicine. 2009; 37(1):58-69. doi: 10.1007/s12160-009-9083-x

[21] Lillis J, Hayes S. Measuring avoidance and inflexibility in weight related problems. International Journal of Behavioral Consultation and Therapy. 2008; 4(4):348-354. doi: 10.1037/h0100865

[22] Berman M, Boutelle K, Crow S. A case series investigating acceptance and commitment therapy as a treatment for previously treated, unremitted patients with anorexia nervosa. European Eating Disorders Review. 2009; 17(6):426-434. doi: 10.1002/ erv.962

[23] Hrabosky J, Cash T, Veale D, Neziroglu F, Soll E, Garner D, et al. Multidimensional body image comparisons among patients with eating disorders, body dysmorphic disorder, and clinical controls: A multisite study. Body Image. 2009; 6(3):155-163. doi: 10.1016/j.bodyim.2009.03.001

[24] Cash T, Santos M, Williams E. Coping with body-image threats and challenges: Validation of the body image coping strategies inventory. Journal of Psychosomatic Research. 2005; 58(2):190-199. doi: 10.1016/j.jpsychores.2004.07.008

[25] Cash T. Body image: Past, present, and future. Body Image 2004; 1(1):1-5. doi: 10.1016/s1740-1445(03)00011-1

[26] Rabiei M, Salahian A, Bahrami F, Palahang H. [Construction and standardization of the body dysmorphic metacognition questionnaire (Persian)]. Journal of Mazandaran University of Medical Sciences. 2011; 21(83):43-52.

[27] Wells A, Matthews G. Modelling cognition in emotional disorder: The S-REF model. Behaviour Research and Therapy. 1996; 34(11-12):881-8. doi: 10.1016/s0005-7967(96)00050-2

[28] Rachman S, Shafran R. Cognitive distortions: thought-action fusion. Clinical Psychology \& Psychotherapy. 1999; 6(2):80-85 doi: 10.1002/(sici)1099-0879(199905)6:2<80::aid-cpp188>3.0.co;2-c

[29] Cartwright-Hatton S, Wells A. Belief about worry and intrusion: the metacognitive Questionnaire. Journal of Anxiety Disorders. 1997; 11(3):279-315. doi: 10.1016/s0887-6185(97)00011-x

[30] Veale D. Advances in a cognitive behavioural model of body dysmorphic disorder. Body Image. 2004; 1(1):113-125. doi 10.1016/s1740-1445(03)00009-3 
[31] Izaadi A, Karimi J, Rahmani M. [Psychometric analysis of Persian version of body image flexibility questionnaire (BI-AAQ) among university students (Persian)]. Hayat. 2013; 19(3):56-69

[32] Jöreskog KG, Sörbom D. LISREL 8.80 for windows (computer software). Lincolnwood, IL: Scientific Software International, Inc; 2006.

[33] Brown T. Confirmatory factor analysis for applied research. New York: Guilford Press; 2006

[34] Schermelleh-Engel K, Moosbrugger H, Müller H. Evaluating the fit of structural equation models: Tests of significance and descriptive goodness-of-fit measures. International Journal of Methods in Psychiatric Research. 2003; 8(8):23-74.

[35] Hair JF, Black WC, Babin BJ, Anderson RE. Multivariate data analysis. Upper Saddle River, NJ: Prentice Hall; 2009.

[36] Hu L, Bentler P. Cutoff criteria for fit indexes in covariance structure analysis: Conventional criteria versus new alternatives. Structural Equation Modeling: A Multidisciplinary Journal. 1999; 6(1):1-55.

[37] Tabachnick B, Fidell L. Using multivariate statistics. Boston: Pearson; 2007. 
